\title{
Mechanical and Electrical Control of Charged Domain Walls in Ferroelectric Materials
}

\author{
L. $\mathrm{Li}^{1}$, J. R. Jokisaari ${ }^{1}$, A. Melville ${ }^{2}$, C. Adamo ${ }^{2}$, D. G. Schlom ${ }^{2}$, and X. Q. Pan ${ }^{1}$ \\ 1. Department of Materials Science and Engineering, University of Michigan, Ann Arbor, MI 48109, \\ United States \\ 2. Department of Materials Science and Engineering, Cornell University, Ithaca, NY 14853, United \\ States
}

Charged domain walls (CDWs) in ferroelectrics, as a result of "head-to-head" or "tail-to-tail" polarization configurations, are of significant scientific and technological importance, as they have been shown to play a critical role in affecting the atomic structures and controlling the electric, photoelectric and piezoelectric properties of ferroelectric materials. An understanding of the local response and underlying dynamic mechanism of CDWs under external excitations is necessary to engineer reliable ferroelectric devices. In this work, we present the nanoscale behavior of individual CDWs under applied mechanical stress and electric fields in $\mathrm{a} \mathrm{BiFeO}_{3}$ thin film using in situ transmission electron microscopy (TEM).

$20 \mathrm{~nm}$ thick $\mathrm{BiFeO}_{3}$ (BFO) film with $20 \mathrm{~nm}$ thick $\mathrm{La}_{0.7} \mathrm{Sr}_{0.3} \mathrm{MnO}_{3}$ (LSMO) bottom electrode were grown on (110) $\mathrm{TbScO}_{3}$ substrates by reactive molecular-beam epitaxy (MBE). CDWs are located either above the triangular $109^{\circ} / 180^{\circ}$ domain wall junction near the top surface (Figure 1a), or below the triangular junction near the bottom interface (Figure 1d). The structures of these two types of CDWs are both different from the rhombohedral-like structure of the bulk BFO domains: the former has a tetragonal-like (T-like) structure, while the latter has a pseudocubic structure with an out-of-plane polarization.[1] As shown in Figure 1a-c, pressing the probe against the film suppresses the T-like structure of CDW near the surface, increases the bound charge there, and thus destabilizes the CDW. For the CDW near the bottom interface (Figure 1d-f), where the bound charge is compensated by the bottom electrode, an applied mechanical stress would compensate the strain between the CDW and surrounding domains, and thus stabilize the CDW and make it wider. As shown in Figure 2, annihilation or formation of the stable CDW can also be observed upon applying a negative (Figure 2a-d) or positive (Figure 2e-h) bias on the probe, respectively, since the induced electric field can enable the motion of the triangular $109^{\circ} / 180^{\circ}$ domain wall junction.

In conclusion, by using in-situ transmission electron microscopy, we have shown that the stability of the charged domain wall in ferroelectric materials can be affected by mechanical stress; we also demonstrated the ability to write and erase stable charged domain walls by applying electric field. Our results provide new insights into understanding of domain walls dynamics of and suggest a route to engineer ferroelectric devices.

\section{References:}

[1]. Li, L., et al., Nano Letters, 2013. 13(11) 5218-5223.

[2]. The authors gratefully acknowledge the financial support through DOE grant DoE/BES DE-FG02-07ER46416. 

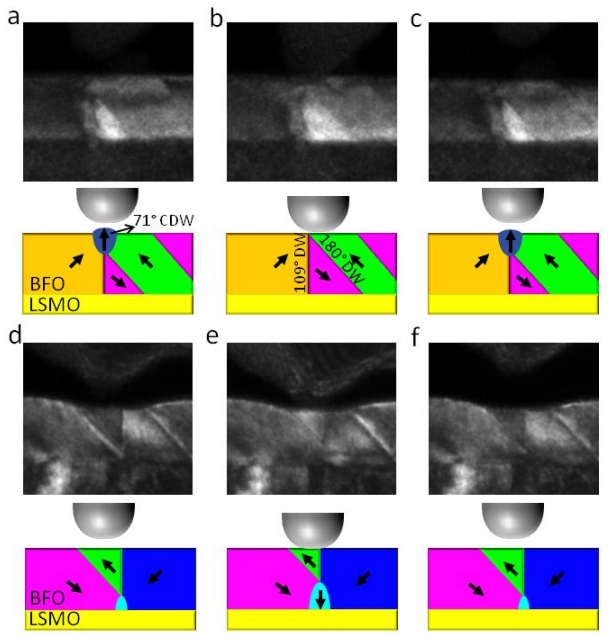

Figure 1. Mechanical control of charged domain walls (CDWs) (a)-(c) Dark-field diffraction contrast TEM images extracted from a video showing the evolution of a CDW near the top surface (a) before, (b) during, and (c) after out-of-plane compression. (d)-(f) Dark-field diffraction contrast TEM images extracted from a video showing the evolution of a CDW near the substrate interface (d) before, (e) during, and (f) after out-of-plane compression. (The scale bar for all TEM images can be extracted from the fact that the BFO film is $20 \mathrm{~nm}$ in thickness)
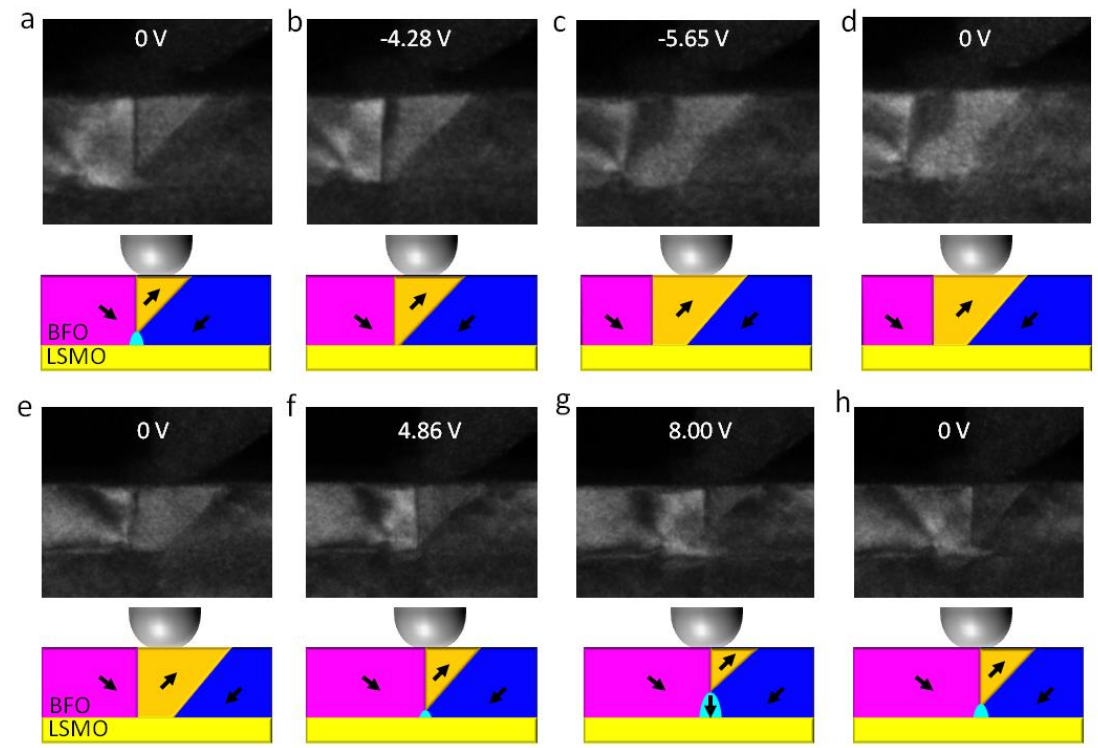

Figure 2. Electrical control of CDWs. (a)-(d) Dark-field diffraction contrast TEM images extracted from a video showing the evolution of a CDW near the substrate interface (a) before, (b)-(c) during, and (d) after a negative bias ramp. (e)-(h) Dark-field diffraction contrast TEM images extracted from a video showing the evolution of a CDW near the substrate interface (e) before, (f)-(g) during, and (h) after a positive bias ramp. 